\title{
WildCARDs: Inflammatory caspases directly detect LPS
}

\author{
Cell Research (2015) 25:149-150. doi:10.1038/cr.2014.128; published online 30 September 2014
}

Inflammasomes are sensors that serve as activation platforms for caspase-1 - a mechanism that set the prevailing paradigm for inflammatory caspase activation. A recent Nature paper by Shi et al. upends this paradigm by describing an unprecedented model for caspase activation whereby caspase $-4,-5$, and -11 directly bind their agonist, cytosolic LPS, triggering auto-activation and subsequent pyroptotic cell death.

The inflammatory caspases - among them caspase- 1 , murine caspase- 11 , and human caspase-4 and -5 (homologs of murine caspase-11) - are central to depriving infectious agents of intracellular replication niches. Upon responding to a given stimulus, they become catalytically active and initiate a form of programmed inflammatory cell death termed pyroptosis. By examining their homology and adjacent chromosomal arrangement in humans and other mammals, it is apparent that the inflammatory caspases originate from a series of gene duplications and subsequent divergences.

A balanced caspase- 1 response is critical to defense against a variety of infectious agents, whereas its aberrant activation underlies a number of immune pathologies. Less is known about caspase-11, -4, and -5 in infection; however, we have shown that one physiological role of caspase- 11 is to detect and help clear cytosol invasive infections, such as those caused by Burkholderia thailandensis [1]. More recent work has shown that caspase-11 mediates resistance to DSS-induced colitis [2] and clearance of Salmonella enterica serovar Typhimurium-infected cells in the intestinal epithelium [3], perhaps limited to the times when these bacteria enter the cytosol. Likewise, caspase-4 responds to $S$. Typhimurium, enteropathogenic E. coli [3], and Shigella flexneri [4] infections in human intestinal epithelial cells. As with caspase-1, moderation of caspase-11 activity is key to limiting immune pathology: much of the lethality of bolus lipopolysaccharide (LPS) injection is mediated by caspase-11 [5-8]. Shedding light on the mechanisms underlying these observations, our lab and that of Dr Vishva Dixit independently determined that caspase-11 is activated in response to cytosolic LPS [7, 8]; however, whether caspase-4 (and/or -5) functions similarly was not determined.

Of the inflammatory caspases, the activation mechanism of caspase- 1 is the best described. Via its N-terminal CARD domain and an adaptor protein called ASC, caspase-1 interacts with a family of cytosolic proteins, the inflammasomes, that detect signatures of infection (Figure 1). It then initiates pyroptosis and directs proinflammatory cytokine secretion. Inflammasomes thus follow the paradigm of apoptotic caspase activation, where apoptosis initiators caspase- $2,-8$ and -9 are recruited and activated by death domain family-containing upstream sensors: the piddosome, DISC, and apoptosome, respectively. Therefore, we and others assumed that the model of upstream sensor activating downstream caspase would hold for the other inflammatory caspases as well. For example, Kayagaki and colleagues coined the term 'noncanonical inflammasome pathway' to describe activation of caspase- 11 by a putative LPS sensor [5, 6]. However, a recent elegant paper by Shi et al. [9] proves this hypothesis wrong and describes an entirely novel paradigm of caspase activation. Moreover, the authors address many of the gaps in our understanding of caspase-11, -4, and -5 biology.

Using electroporation to deliver bacterial components into the cytosol of cells, the authors first determined that caspase-4 responds to LPS in human monocytes by triggering pyroptosis. These findings extended to non-myeloid cells, where caspase- 4 is constitutively expressed. The authors then demonstrated that caspase- 4 and caspase- 11 are functionally interchangable, supporting that they are homologs.

Shi and colleagues next began identifying the molecule that actually binds LPS in the cytosol. They screened a number of NLRs and CARD domaincontaining proteins, but no candidates emerged. In agreement with this, unpublished work from our lab also ruled out virtually all known CARD-containing proteins as the LPS sensor. Clues to the identity of the sensor arose from the following astute observations: First, Shi et al. noticed that both caspase-4 and caspase-11, when purified from E. coli, eluted from columns as large oligomers, suggesting activation, whereas they eluted as monomers when expressed in and purified from insect cells. Second, they found that the LPS contents of caspase- 4 and -11 purified from $E$. coli were three orders of magnitude higher than what they typically observed when purifying bacterial proteins. Together, these results suggested that caspase- 4 and -11 directly bind LPS. A series 


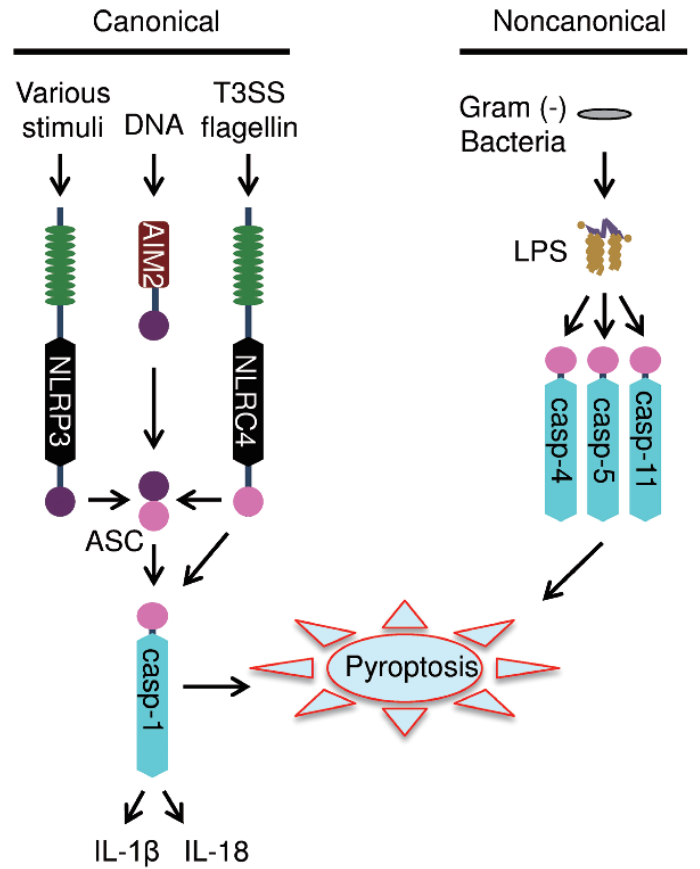

of pull-downs and surface plasmon resonance experiments confirmed this notion, revealing stable interaction of LPS with caspase-4 and -11 in cells transfected with LPS. Furthermore, the authors showed that caspase- 5 similarly binds LPS. In all cases, LPS binding and caspase oligomerization was CARD domain dependent; indeed, purified caspase- 4 and -11 CARD domains were sufficient to bind LPS and oligomerize. Three regions of basic residues in the caspase-11 CARD domain - mostly conserved in caspase- 4 and -5 , but not caspase-1 - were critical for LPS binding. Last, the authors determined that caspase- 11 and -4 oligomerization stimulates activation, as measured by cleavage of a fluorogenic substrate. Interestingly, the known antagonists of caspase-11 activation Lipid IVa and atypical LPS from Rhodobacter sphaeroides bound caspase-4 and -11, but failed to induce oligomerization and activation.

The Shi et al. paper brings to light a number of fascinating perspectives. First, binding of LPS by caspase- $4,-5$,
Figure 1 Schematic of canonical and noncanonical inflammasome pathways for inflammatory caspase activation. Left: Inflammasomes such as AIM2, NLRP3, and NLRC4 detect contamination of the cytosol with microbial ligands (e.g., DNA, flagellin, bacterial type 3 secretion system components) or certain cellular perturbations. Via the adaptor protein ASC, they subsequently activate caspase-1 (in the case of certain CARDcontaining inflammasomes, such as NLRC4, direct interaction with caspase-1 can also occur), which initiates pyroptosis and secretion of the proinflammatory cytokines IL-1 $\beta$ and IL-18. Right: Caspase-4, -5 , and -11 directly bind cytosolic LPS from Gram-negative bacteria. They subsequently oligomerize, activate, and initiate pyroptosis. and -11 establishes a new paradigm for caspase activation: Direct detection of a cell death-inducing ligand by a caspase. As the authors noted, this is analogous to horseshoe crab factors $C$ and $G$, which bind LPS and $\beta-(1,3)-D-$ glucan, respectively, and initiate coagulation cascades in haemolymphs.

Second, the cell expression patterns of caspase-11, -4, and -5 may have important implications in future strategies for treating endotoxemia and Gramnegative sepsis. Caspase-11 expression is inducible in myeloid cells, where its basal expression is low; in contrast, caspase- 4 appears to be constitutively expressed in human myeloid cells. Therefore, aberrant translocation of LPS into the cytosol of human myeoloid cells may not require priming to activate caspase- 4 and initiate pyroptosis, perhaps sensitizing humans to the deleterious effects of LPS compared to mice. Investigating other cell type expression differences in this context will be informative.

In answering so many questions about the biology of the inflammatory caspases, the work of Shi and colleagues raises many more. Among them: Why do antagonists of caspase-11 fail to induce oligomerization? How do the CARD domains of these caspases "see" LPS? During binding of LPS by MD2, the acyl chains of lipid A extend into the binding cleft of MD2 [10] in a manner sensitive to acyl chain length; in contrast, caspase-11 detects very diverse lipid A acyl chain lengths and structures, such as those of Salmonella and Legionella species [7, 8, 11], suggesting that the CARD domain may wrap around the lipid groups of LPS near the phosphate head groups of lipid A. Insights into these questions will surely come from crystal structures of caspase- $11,-4$, and -5 bound to various LPS structures.

Jon Alan Hagar ${ }^{1}$, Youssef Aachoui ${ }^{1}$, Edward Axel Miao ${ }^{1}$

${ }^{1}$ Department of Microbiology and Immunology and Lineberger Comprehensive Cancer Center, University of North Carolina at Chapel Hill, Chapel Hill, NC 27599, USA

Correspondence: Edward Axel Miao

E-mail: emiao@med.unc.edu

\section{References}

1 Aachoui Y, Leaf I, Fontana M, et al. Science 2013; 339:975-978.

2 Demon D, Kuchmiy A, Fossoul A, et al. Mucosal Immunol 2014; 7:1480-1491.

3 Knodler LA, Crowley SM, Sham HP, et al. Cell Host Microbe 2014; 16:249-256.

4 Kobayashi T, Ogawa M, Sanada T, et al. Cell Host Microbe 2013; 13:570-583.

5 Wang S, Miura M, Jung YK, et al. Cell 1998; 92:501-509.

6 Kayagaki N, Warming S, Lamkanfi M, et al. Nature 2011; 479:117-121.

7 Kayagaki N, Wong MT, Stowe IB, et al. Science 2013; 341:1246-1249.

8 Hagar JA, Powell DA, Aachoui Y, et al. Science 2013; 341:1250-1253.

9 Shi J, Zhao Y, Wang Y, et al. Nature 2014; 514:187-192.

10 Park BS, Song DH, Kim HM, et al. Nature 2009; 458:1191-1195.

11 Pilla DM, Hagar JA, Haldar AK, et al. Proc Natl Acad Sci USA 2014; 111:6046-6051. 